\title{
Gut microbiota maturation during early human life induces enterocyte proliferation via microbial metabolites
}

\author{
Michael W. Dougherty ${ }^{1}$, Oleksandr Kudin², Marcus Mühlbauer ${ }^{1}$, Josef $\mathrm{Neu}^{2}$, Raad Z. Gharaibeh ${ }^{1}$ and \\ Christian Jobin ${ }^{1,3^{*}}$ (iD
}

\begin{abstract}
Background: The intestinal tract undergoes a period of cellular maturation during early life, primarily characterized by the organization of epithelial cells into specialized crypt and villus structures. These processes are in part mediated by the acquisition of microbes. Infants delivered at term typically harbor a stable, low diversity microbiota characterized by an overrepresentation of various Bacilli spp., while pre-term infants are colonized by an assortment of bacteria during the first several weeks after delivery. However, the functional effects of these changes on intestinal epithelium homeostasis and maturation remain unclear. To study these effects, human neonate feces were obtained from term and pre-term infants. Fecal 165 rDNA sequencing and global untargeted LC-MS were performed to characterize microbial composition and metabolites from each population. Murine enteral organoids (enteroids) were cultured with $0.22 \mu \mathrm{m}$ filtered stool supernatant pooled from term or pre-term infants.
\end{abstract}

Results: Term and pre-term microbial communities differed significantly from each other by principle components analysis (PCOA, PERMANOVA $p<0.001$ ), with the pre-term microbiome characterized by increased OTU diversity (Wilcox test $p<0.01)$. Term communities were less diverse and dominated by Bacilli (81.54\%). Pre-term stools had an increased abundance of vitamins, amino acid derivatives and unconjugated bile acids. Pathway analysis revealed a significant increase in multiple metabolic pathways in pre-term samples mapped to E. coli using the KEGG database related to the fermentation of various amino acids and vitamin biosynthesis. Enteroids cultured with supernatant from pre-term stools proliferated at a higher rate than those cultured with supernatant from term stools (cell viability: $207 \%$ vs. 147.7\%, $p<0.01$ ), grew larger (area: $81,189 \mu \mathrm{m}^{2}$ vs. $41,777 \mu \mathrm{m}^{2}, p<0.001$ ), and bud at a higher rate $(6.5$ vs. $4, p<0.01)$. Additionally, genes involved in stem cell proliferation were upregulated in pre-term stool treated enteroid cultures (Lgr5, Ephb2, Ascl2 Sox9) but not term stool treated enteroids.

Conclusions: Our findings indicate that microbial metabolites from the more diverse gut microbiome associated with pre-term infants facilitate stem cell proliferation. Therefore, perturbations of the pre-term microbiota may impair intestinal homeostasis.

Keywords: Bacteria, Intestine, Network, Epithelial cells, Stem cells, Bile acid, Metabolism

\footnotetext{
* Correspondence: Christian.Jobin@medicine.ufl.edu

'Department of Medicine, Division of Gastroenterology, University of Florida, CGRC, 2033 Mowry Rd, Florida 32610, USA

${ }^{3}$ Department of Infectious Diseases and Pathology, College of Veterinary

Medicine, University of Florida, Gainesville, Florida, USA

Full list of author information is available at the end of the article
}

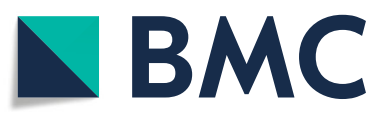

(c) The Author(s). 2020 Open Access This article is licensed under a Creative Commons Attribution 4.0 International License, which permits use, sharing, adaptation, distribution and reproduction in any medium or format, as long as you give appropriate credit to the original author(s) and the source, provide a link to the Creative Commons licence, and indicate if changes were made. The images or other third party material in this article are included in the article's Creative Commons licence, unless indicated otherwise in a credit line to the material. If material is not included in the article's Creative Commons licence and your intended use is not permitted by statutory regulation or exceeds the permitted use, you will need to obtain permission directly from the copyright holder. To view a copy of this licence, visit http://creativecommons.org/licenses/by/4.0/. The Creative Commons Public Domain Dedication waiver (http://creativecommons.org/publicdomain/zero/1.0/) applies to the data made available in this article, unless otherwise stated in a credit line to the data. 


\section{Background}

Shortly after birth, bacteria from the environment colonize the naïve gastrointestinal tract $[1,2]$. In term infants, a low diversity microbiota harboring high proportions of Bifidobacteria (after vaginal birth) or Staphylococcus (after cesarean section) are typically observed shortly after delivery $[3,4]$. In pre-term infants, microbiota maturation follows a ubiquitous pattern characterized by increased diversity and temporally distinct phases with an initial abundance of Bacilli followed by increased proportions of Gammaproteobacteria [5]. The characteristics of initial bacterial communities are closely linked to the maternal microbiome, suggesting colonization occurs during or shortly after birth with subsequent diversification resulting from environmental factors $[6,7]$.

This period of microbial acquisition represents a critical time in terms of development for the infant, and disruption of healthy microbiota acquisition may have acute or far-reaching pathological consequences such as necrotizing enterocolitis (NEC), late onset sepsis, and neurological disorders [8-11]. The presence of specific microbial species may have protective effects on the host. Bifidobacteria spp. maintain gut barrier function by preserving claudin 4 localization at tight junctions, and Clostridia are capable of modulating immune response to dietary antigens preventing allergic responses $[12,13]$. Lactate synthesized by early colonizing microbes in the healthy neonatal intestine may serves as an energy resource for cells via recognition by Gpr81 receptors, subsequent oxidization to pyruvate, and TCA cycle incorporation [14, 15]. Lactate is also capable of promoting stem cell proliferation via Gpr81 receptors and increased WNT activity in adjacent Paneth cells [16].

In addition to the specific influence of particular microbes and associated metabolites, more diverse microbial communities may more efficiently fill functional niches independent of specific species composition. Gut bacteria enzymatically transform and metabolize undigested dietary components and host molecules consequently producing novel metabolites which, in part, mediate these effects [17]. Twin studies have shown microbial communities perform a core set of metabolic functions primarily providing sources of energy for the intestinal epithelium independent of differences in microbiota composition [18]. Bacteria ferment dietary carbohydrates and subsequently synthesize short chain fatty acids, which are preferentially utilized by the gut epithelium as source of energy and also have immunoregulatory functions [19]. A diverse array of common gut bacteria synthesize essential amino acids that serve as necessary precursors to cellular replication [20].

Given this evidence, we hypothesized that the distinct microbial communities acquired by term and pre-term neonates will generate distinct metabolomic profiles in the developing small intestine. In this study, we use small intestinal organoid models to quantify how microbial metabolites from each cohort affect proliferation and differentiation in the intestinal epithelium. We found that term infant microbial communities are characterized by a lack of diversity relative to pre-term infants. Fecal metabolomic profiles from these communities is also altered, with preterm infant fecal metabolomics profiles characterized by increased signatures of amino acid metabolism, vitamin metabolism, and microbially transformed metabolites such as secondary bile acids. Soluble factors present in preterm stool supernatants induce stem cell proliferation in organoid models. Taken together, this evidence suggests microbiota diversification in human neonates may facilitate intestinal proliferation.

\section{Results}

The pre-term infant gut microbiota is more diverse than the term gut microbiota

To study the interaction between host-bacteria associated with term and pre-term delivery, we first performed 16S rRNA gene sequencing to determine fecal microbial composition of neonates from each cohort. Two term samples showed very few $16 \mathrm{~S}$ reads and were removed. Beta diversity analysis using principal coordinate analysis (PCoA) showed that bacterial communities differed significantly in their composition at both the ASV and family levels (Fig. 1a-b). Pre-term microbiome composition was characterized by increased alpha diversity (Shannon: $p=0.008$, Simpson: $\mathrm{p}=0.008$; Fig. $1 \mathrm{c}-\mathrm{d}$ ) and beta diversity $(p=0.0084)$ compared to term composition.

Linear discriminant analysis was performed to determine the significantly enriched bacteria in each cohort. Pre-term communities were characterized by increased ASVs from various bacterial groups, including Clostridia, Enterobacteriaceae, Veillonellaceae, Enterococcaceae, Tissierellaceae, Klebsiella, Vagococcus and Finegoldia (Fig. 1e). Term infant microbial communities were dominated primarily by a single class of bacteria: Bacilli (4 of the 5 samples), or Bacteroidia (one of the 5 samples) (Fig. 1f). In pre-term infants, Gammaproteobacteria was the most common dominant taxa, followed by Clostridia and Bacilli (Fig. 1f). Streptococcus and Staphylococcus were the most abundant families in term stool samples (Fig. 1g). The pre-term microbiota was more diverse, with larger proportions of Enterobacteriaceae and Veillonellaceae, and lower relative abundances of early colonizers such as Streptococcus (Fig. 1g).

The pre-term neonate metabolome is characterized by an increase in bacterially transformed products, vitamins, and amino acid derivatives

To examine how these distinct microbial communities may functionally affect the host intestinal development, 


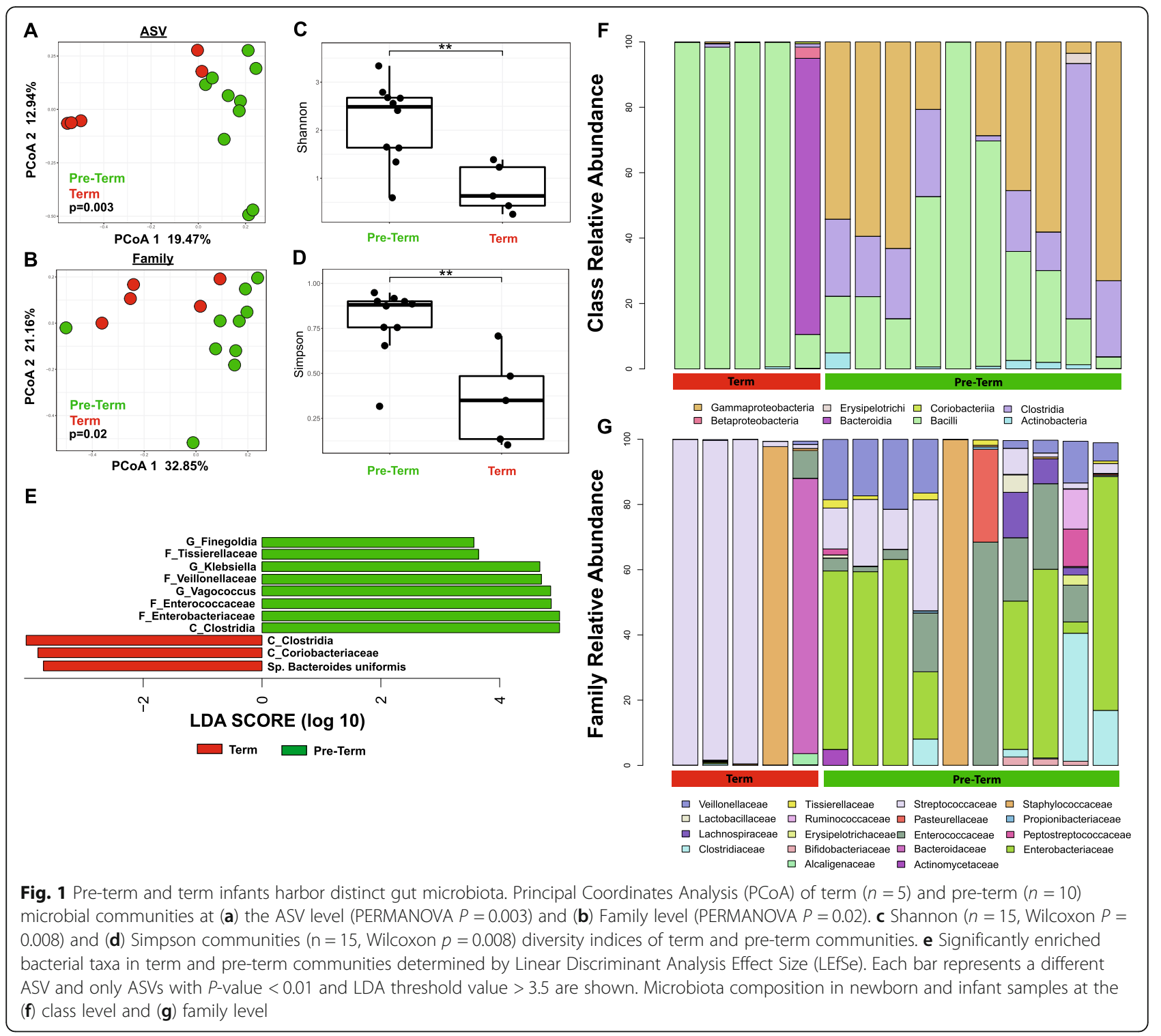

we performed global untargeted LC-MS and metabolomics on a subset of stools used in the 16S rRNA sequencing. A total of 14,342 metabolites were detected using positive and negative ion modes, and 374 metabolites were positively identified (Supplemental Tables 1-2), of which 48 metabolites were significantly altered between term and pre-term infants (Supplemental Table 3). Principal component analysis (PCA) showed significant differences in total metabolic composition (Fig. 2a) as well as composition of identified metabolites (Fig. 2b) with age at delivery. Similarly, metabolomics profiles of fecal samples from each cohort were highly correlated with each other (Fig. 2c).

Pre-term fecal samples exhibited metabolic profiles with a higher relative abundance of various vitamins, amino acids, or byproducts of amino-acid metabolism, while term fecal samples contained higher relative abundances of conjugated bile acids and intermediaries of the TCA cycle (Fig. 2d). Pre-term samples were classified by an increase in several bacterial-associated metabolites involved in amino acid metabolism (histamine, putrescine), bacterial-derived vitamins and associated enzymes (pantothenic acid, pantetheine), and a decrease in the primary bile acids glycodeoxycholic acid (GCDCA) and tauroursodeoxycholic acid (TUDCA; Fig. 2e). An increase in conjugated bile acids was observed in the term metabolome, concurrent with an increase in bacterially transformed bile acids in pre-term samples indicating an interaction between microbes and luminal host metabolites (Fig. 2f). However, metabolomics analysis was unable to differentiate between deoxycholic acid and chenodeoxycholic acid, and individual sample variation 


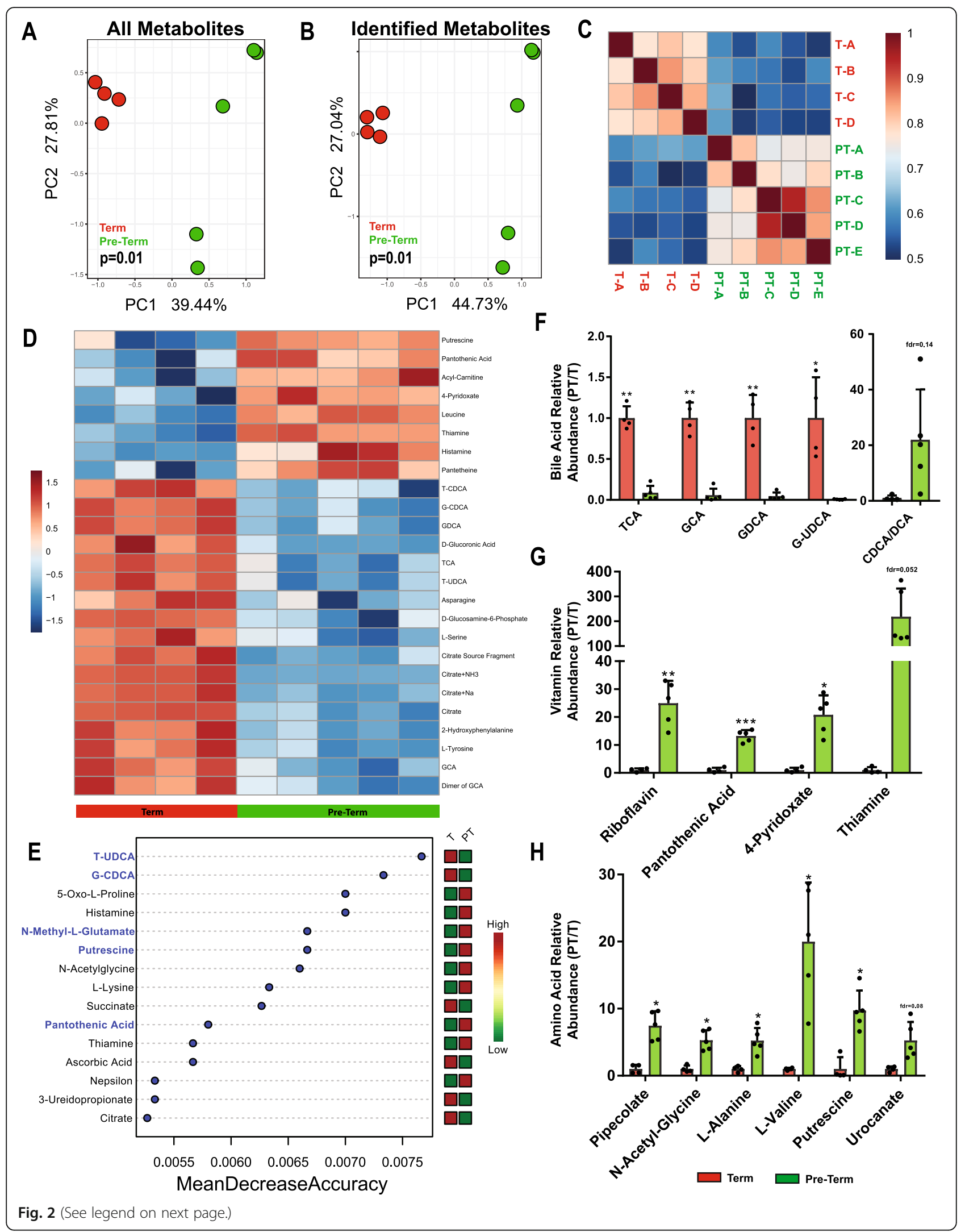


(See figure on previous page.)

Fig. 2 Distinct metabolomics profiles are associated with term and pre-term microbial communities. Principal component analysis (PCA) of term $(n=4)$ and pre-term $(n=5)$ infants listing $(\mathbf{a})$ all metabolites (PC1 Wilcox $P=0.01)$ and $(\mathbf{b})$ positively identified metabolites $(P C 1$ Wilcox $P=0.01)$. $\mathbf{c}$ Heatmap visualizing correlations between identified metabolomics profiles. $\mathbf{d}$ Heatmap visualizing the top- 25 most significantly enriched metabolites in term and pre-term metabolomics profiles. e Random forest identification of top 15 differentially abundant metabolites in term and pre-term metabolomics profiles, blue text identifies microbially synthesized or bio-transformed compounds. Difference in specific metabolite concentrations determined using Wilcoxon rank sum tests for $(\mathbf{f})$ bile acids, $(\mathbf{g})$ vitamins, and $(\mathbf{h})$ amino acids. All $P$ values are Benjamini-Hochberg corrected, ${ }^{*}=\mathrm{fdr} P<0.05,{ }^{* *}=\mathrm{fdr} P<0.01$. Metabolomics data is represented as the mean of three technical replicates. For all panels:

$\mathrm{T}=$ term, $\mathrm{PT}=$ pre-term

in these concentrations was high (Fig. 2f). Additionally, the pre-term metabolome had a higher relative abundance of various vitamins and amino acid derivatives (Fig. 2g-h).

These results show that increased microbiota abundance and diversity is associated with changes to overall fecal metabolomics profiles, and suggests that the microbiota may modulate molecules in the intestine by biotransformation of primary bile acids, fermentation of dietary amino acids, and vitamin biosynthesis.

\section{The neonatal intestinal microbiota influences gut metabolites}

Analysis at the class level showed the dominant classes from pre-term samples (Clostridia and Gammaproteobacteria) were correlated with an increase of several microbeassociated metabolites (Fig. 3a), while the dominant class from term samples (Bacilli) correlated with a decrease of several microbe-associated metabolites (Fig. 3a). Higher abundance in three pre-term enriched families (Enterobacteriaceae, Enterococcaceae, and Tissierellacea) correlated with an increase of several microbially associated metabolites implicated in promoting proliferation in organoid models [21] (e.g. nicotinate, sarcosine, pyridoxate/ pyridoxamine, succinate, uracil; Fig. 3b). Additionally, an increase in metabolites related to proliferation via amino acid synthesis or metabolism were correlated with these bacterial families (e.g. Ureidopropionate; Fig. 3b). Taken together, these data suggest that potential functional changes occur as the microbiome diversifies during early life, possibly through biosynthesis of bacterial metabolites or modulation of dietary/host-derived metabolites such as primary bile acids.

In order to understand how bacterial/host biosynthesis or transformation of metabolites shaped the metabolome, we identified enriched metabolic pathways in infant samples using KEGG (Kyoto Encyclopedia of Genes and Genomes) pathways of the model bacteria Escherichia coli and the human host, Homo sapiens. When metabolomics data was mapped to $E$. coli, a significant increase in 8 pathways was detected in pre-term neonates (Fig. 4a). Upregulated pathways in these microbial communities primarily related to amino acid metabolism, and biosynthesis of essential vitamins (e.g. pantothenic acid) and amino acids (e.g. lysine) (Supplemental Table 4). When mapped to H. sapiens, 20 significantly enriched pathways predominately related to the metabolism of various amino acids and vitamins (Fig. 4b, Supplemental Table 5). Degradation of the essential amino acid lysine was increased in conjunction with an increase in lysine biosynthesis by bacteria, demonstrating potential host utilization of microbial metabolites (Fig. 4a-b). Similarly, upregulation of human histidine metabolism was observed in pre-term samples, likely accounting for increased urocanate (a derivative of l-histidine) concentrations (Supplemental Table 5).

\section{Metabolites from pre-term microbial communities increase enterocyte proliferation}

To examine the potential effects of neonatal gut metabolites on enterocyte development, murine enteroids were cultured with pooled filtered stool supernatants obtained from human term and pre-term neonates previously analyzed by metabolomics. Cellular proliferation was measured by MTT assay, and results showed an increase in cell abundance after 3- and 5-days treatment with preterm stool supernatans (Fig. 5a). Enteroids cultured with pre-term stool supernatant grew more rapidly than term or control enteroid cultures, exhibiting a more robust phenotype after 5-days of culture indicating more efficient growth and maturation (Fig. 5b-d). Additionally, enteroids treated with pre-term stool supernatants formed crypt domains earlier and at a higher rate than term-supernatant and control treated enteroids (Fig. 5d). Enteroids cultured with pre-term stool supernatant also exhibited increased Ki67 localized primarily to the base of newly budding crypts (Fig. 5e,f). Collectively, these data suggest that metabolites present in stool samples harboring more abundant and diverse microbiota induce enterocyte proliferation.

In addition, we observed increased $L g r 5, A s c l 2$, and Ephb2 mRNA expression in pre-term treated enteroids, which are markers of stem cell proliferation, and concomitant increase in Wnt signaling genes Sox9 and Fzd5 [22] (Fig. 5g). No difference was observed in markers of differentiation towards other enterocyte lineages ( $L y z 1$, Muc2, Chga). Pre-term stool supernatant treatment also slightly downregulated markers of ER stress and 


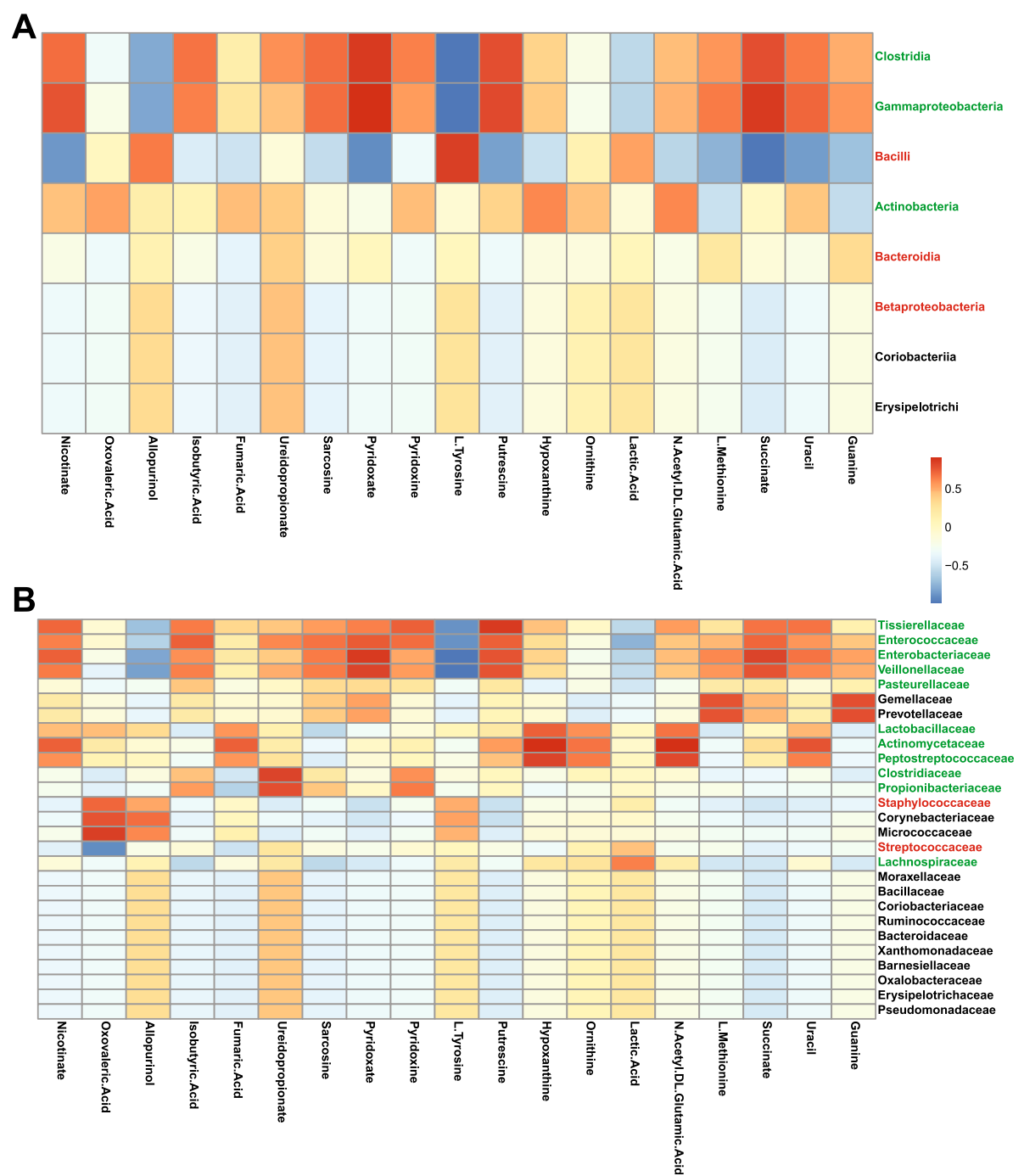

Fig. 3 Correlation between known microbial metabolites linked to proliferation and microbiota composition. Heatmap visualizing Pearson's correlation coefficients relating relative metabolite concentrations with relative bacterial abundance at (a) the class and (b) family level. Red text denotes bacterial taxa associated with term $(n=4)$ microbiota, green text denotes bacterial taxa associated with pre-term $(n=5)$ microbiota

inflammation (CHOP, TNFa, IFNy; Fig. 5h). Taken together, this evidence suggests that the proliferative phenotype is driven by stem cell niche expansion in response to microbial metabolites isolated from neonate stool harboring a diverse microbiome.

\section{Discussion}

Establishment of a healthy microbiome in infants is essential for normal growth and development of intestine, optimal metabolic function as well as maturation of the immune system [17, 23, 24]. Therefore, altered microbiota composition puts infants at risk for both shortand long-term health implications. In healthy infants, the intestine is typically colonized by lactic acid bacteria (LAB) derived from breast milk [25]. Enteroids cultured with Lactobacillus exhibit increased Ki67 expression, and adding lactate to the culture increases stem cell proliferation by increasing WNT production in adjacent Paneth cells $[16,26]$. Given the increased LAB abundance in the term composition we expected to observe a difference in lactate level between our samples and increased epithelial proliferation in enteroids treated with term stool supernatants [16]. Interestingly, lactate levels between term and pre-term samples were not different, possibly because the host utilizes these metabolites prior to excretion, and no increased proliferation was observed. Because of this, the lack of a proliferative phenotype in enteroids treated with term infant stool supernatants may suggest that intestinal maturation in healthy infants is driven by key microbes and metabolites missing in our stool supernatants rather than the robust function of a complex community. 

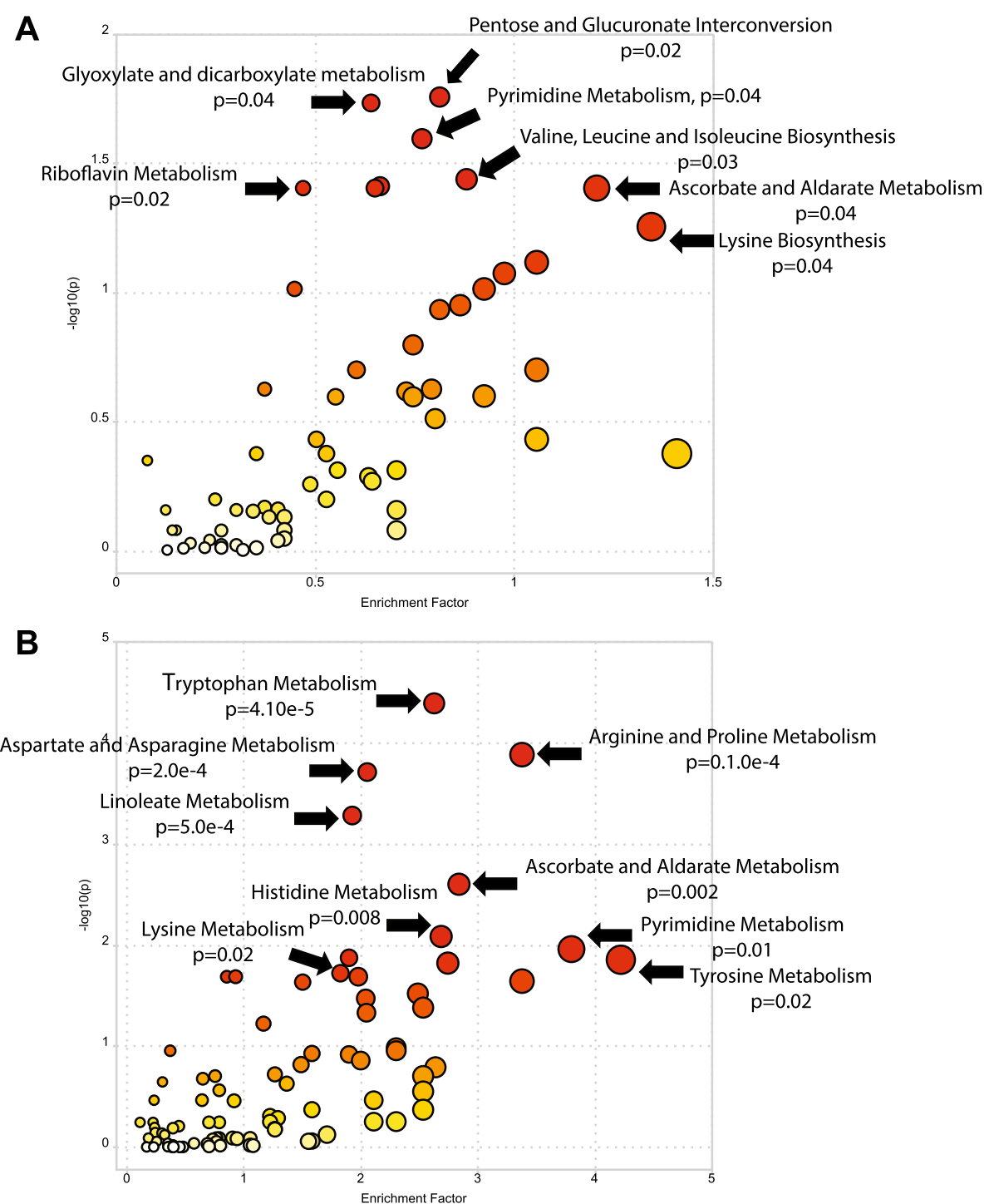

Fig. 4 KEGG metabolomic pathway analysis showing significantly enriched pathways in pre-term infant cohort. Metabolic pathways enriched in PT samples mapped to Homo sapiens (a) and Escherichia coli (b), with the most significantly enriched pathways identified; $P$ values under each pathway's name denote enrichment of entire pathway by Fisher's exact test

Alternatively, epithelial proliferation may increase through the total metabolic output of a complex microbial community which more effectively shapes the total metabolomics profile. Several studies have shown that mice harboring a complex microbiota exhibit higher rates of proliferation and tissue growth than germ free counterparts, a phenomenon which may result from editing of metabolomic profiles by the microbiota $[27,28]$. Gut microbes perform a set of core metabolic functions [18], pathways related to core microbial metabolic functions (pyrimidine metabolism, amino acid metabolism, and the biosynthesis of secondary metabolites) were significantly upregulated in our pre-term supernatant samples. Treatment with pre-term stool supernatant increased organoid proliferation accordingly, suggesting soluble factors in the stool mediate these effects. Increased absolute cell numbers demonstrate that these changes derive from increased cell cycling, rather than increased luminal contents or cell distension. Furthermore, mRNA expression profiles are consistent with increased stem cell proliferation and WNT signaling pathways. Notably, organoids increased in size and crypt domains rather than forming "cystic" structures typical of undifferentiated stem cells. One could hypothesize that the proliferative effect of pre-term gut metabolites observed in our study may serve a protective role for the epithelium. Perturbations of these communities (e.g. exposure to antibiotics) and concurrent dysbiosis may limit these effects and put pre-term infants at risk for disease. 


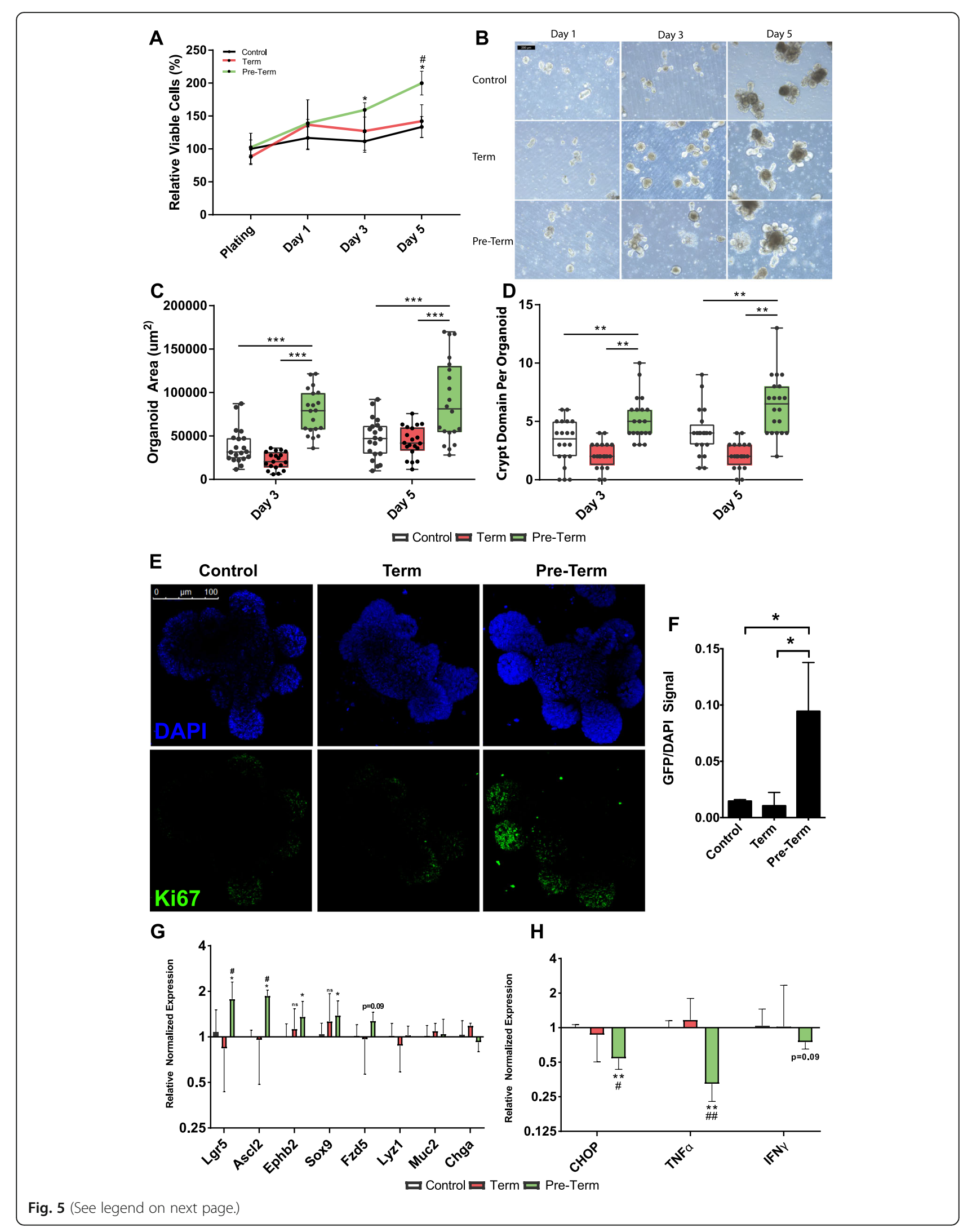




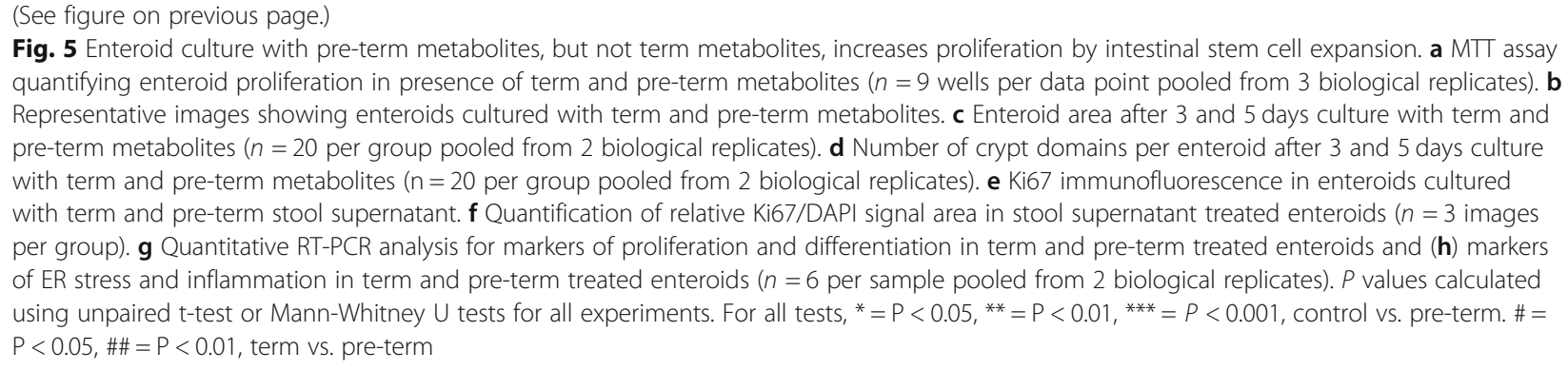

Determining which specific metabolites and hostmicrobe dynamics from our pre-term stool supernatants increase proliferation may have therapeutic potential. In our study, the more diverse pre-term microbiota was characterized by metabolomics profiles with a significant increase of amino acid derivatives, such as putrescine. Putrescine is a polyamine, a class of molecules which serve as a precursor to cellular division whose concentrations are particularly high in rapidly proliferating tissue such as the small intestine $[29,30]$. Putrescine is involved in nucleic acid and protein synthesis during cellular proliferation, preceded by the generation of putrescine by decarboxylation amino acids such as arginine and ornithine [31, 32]. Putrescine itself is synthesized by a number of bacterial species and has been shown to slightly increase proliferation in organoids [21, 33, 34]. In our study, both ornithine and putrescine abundances correlated to specific bacterial groups characterizing the pre-term microbiome including Clostridia, Gammaproteobacteria, and Actinobacteria. Additionally, urocanate and histamine were more abundant in pre-term samples, coupled with increased histidine metabolism. Urocanate and histamine are both byproducts of l-histidine metabolism, and l-histidine is an essential amino acid biosynthesized by many bacterial species [35]. L-histidine deficiency has been shown to induce mitochondrial dysfunction in the intestinal epithelium, and histamine receptor agonists increase epithelial proliferation in the gut $[36,37]$. The increased urocanate and histamine concentrations in pre-term metabolomics profiles suggest increased deamination of 1-histidine in the presence of a complex microbiota, a phenomenon that may enhance epithelial viability and mitochondrial function. Taken together, this evidence suggests the microbiota may supply amino acids necessary for cellular proliferation, which may increase stem cell cycling and viability rate. However, formula and breast milk generally contain amino acids and thus it remains unclear if amino acid biosynthesis by bacteria is an essential component of development or whether the host receives adequate quantities of essential metabolites from the diet.

Bile acid deconjugation was significantly increased in the pre-term metabolomes, yielding high deoxycholic acid (DCA) concentrations. Lower concentrations of primary bile acids in pre-term samples were coupled with an increase in primary bile acid biosynthesis suggesting that pre-term microbial communities are efficiently biotransforming host products to DCA [38]. A large majority of bile acids are reabsorbed by the ileum, and DCA is of particular interest due to its hydrophobic nature and increased permeability through the small intestine [39]. Once reabsorbed, bile acid derivatives play a key role in modulating microbial communities by acting on Farnesoid X receptors (FXRs) present on multiple epithelial lineages including Lgr5+ stem cells [40, 41]. Recent evidence suggests FXR activation regulates proliferation by inhibiting stem cell expansion, and that DCA exposure counters this inhibition by blocking FXR gene targets and activating EGFR signaling pathways [42-45]. EGFR signaling is a crucial component of stem cell niche maintenance in the gut. EGF and EGF-like growth factors have been shown to increase intestinal stem cell proliferation and protect against anoxia induced stem cell apoptosis and bacterial translocation in both mouse models and cell lines [46]. Furthermore, organoid models exposed to DCA exhibit increased expression of WNT pathway genes and stem cell markers [42]. During wound healing, crypt DCA concentrations greatly increase as FXR activation facilitates stem cell regeneration by inhibiting PGE2 expression, and DCA has been correlated with sustained remission in pediatric Crohn's disease $[47,48]$. Taken together, these findings suggest that bacterial transformation of conjugated cholic acids to DCA may play a protective role in the developing preterm gut, driving stem cell proliferation during early life. More work is necessary to determine how various concentrations and temporal patterns of DCA exposure may regulate intestinal maturation throughout early life.

Epithelial maturation facilitated by microbial colonization has been well defined in preclinical mouse models. Here we provide evidence that similar phenomenon occur in human neonates during early life. Functionally, increased proliferation and tissue maturation is an essential developmental process particularly in pre-term infants, and increased proliferation can be beneficial during early life as a method of overcoming stem cell disfunction resulting from 
endoplasmic recticulum stress [49] or mitochondrial dysfunction [50]. Similarly, microbially-induced stem cell proliferation supports appropriate crypt-villus architecture and formation of deeper progenitor zones which may support epithelial health by protecting intestinal stem cells from microbial interaction [21, 27].

A limitation of our analyses is the inability to decouple microbial and host-derived metabolites, which we can only achieve through a priori knowledge. Furthermore, untargeted metabolomics is limited by the available metabolite library and generally only provides a small number of confirmed metabolite identities. Thus, identity limitations may obfuscate metabolites inducing proliferative phenotypes. Metabolite screening by Kaiko et al. has shown a large number of microbial metabolites, including some discussed here, only slightly enhance organoid proliferation when monoassociated in vitro [21]. Given the more robust proliferation response we observed, this suggests there may be an additive or complementary effects when considering the functional effects of entire microbial communities. Additionally, specific metabolites present in the term gut may not be present in fecal samples or in the processed stool supernatant, thus it may not be possible to conclude that microbiotainduced proliferation is strictly limited to pre-term bacterial communities. For example, microbial communities in breast-fed term infants are typically characterized by a long period of low diversity concurrent with increases in overall bacterial abundance and normal epithelial maturation. Thus, this work primarily highlights the potential for the pre-term gut microbiota to induce proliferation and the potential detrimental effects stemming from dysbiosis during this critical window.

\section{Conclusions}

The evidence presented here demonstrates the proliferative effect of pre-term metabolomes on small intestinal organoids. We employ metabolomics analysis to gain insight into potential regulators of proliferation during early life, laying a foundation for further research analyzing the role of microbially derived metabolites in developmental processes. While many of the individual compounds highlighted here have been shown to have marginally proliferative effects on epithelial organoids, our study suggests potential additive effects derived from entire microbial communities. Future studies should focus on defining the functional impact of metabolic pathways outlined here in pathological situation such as necrotizing enterocolitis.

\section{Methods}

\section{Preparation of stool supernatant}

Stool samples were collected from human infants from the neonatal intensive care unit (NICU) and the newborn nursery. Term samples $(n=7)$ were collected from term stool/meconium and pre-term samples $(n=$ 10) were collected from pre-term stool. Pre-term samples were obtained from infants undergoing routine care in the NICU, often characterized by compulsory antibiotic treatment during the first several days of life. Samples were stored briefly at $-20^{\circ} \mathrm{C}$ before being transferred on dry ice and placed at $-80^{\circ} \mathrm{C}$ for longterm storage. Stool samples $(1 \mathrm{~g})$ were suspended in sterile PBS, dissociated by pipetting with a wide bore tip and vortexed vigorously for $30 \mathrm{~s}$ then centrifuged and the supernatant was passed through a $0.22 \mu \mathrm{m}$ filter before storage at $-80^{\circ} \mathrm{C}$.

\section{$16 \mathrm{~S}$ rRNA gene sequencing}

DNA was extracted from neonate stool samples (term: $\mathrm{n}=7$, pre-term: $\mathrm{n}=10$ ) using the Powerlyzer Powersoil DNA isolation kit (Qiagen 12855) according to manufacturer's specifications with slight modifications. The V1V3 hypervariable region of the 16S rRNA gene was amplified using primer pair 8F (5' - AGAGTTTGATCCTG GCTCAG - 3') and 534R (5'-ATTACCGCGGCTGC TGG-3') as previously described [51]. Briefly, forward and reverse primers contained universal Illumina pairedend adapter sequences with unique barcodes between the PCR primer sequence and the Illumina adapter sequence to allow multiplex sequencing. Equimolar amounts of samples were then pooled and sequenced with an Illumina MiSeq.

\section{Analysis of $16 \mathrm{~S}$ rRNA sequences}

Sequencing reads were preprocessed using Quantitative Insights into Microbial Ecology version 2 (QIIME2) [52] including trimming, filtering at Q20 and pair merging. The final set of reads was fed to the DADA2 algorithm within QIIME2 pipeline to infer exact amplicon sequence variants (ASVs) with trim length set to 200 (the average sequence length of the dataset) [53]. Two newborn samples showed very few read count (less than 20 reads) and therefore were removed from subsequent analyses. The remaining samples contained an average of 51,885 reads per sample $(\min =33,516$ reads; $\max =81,138$ reads $)$ incorporated in ASVs. Taxonomic assignment was done using QIIME2's feature-classifier classify-sklearn after training the classifier on the Greengenes 97\% reference dataset (release 13_8). Only ASVs with bacterial taxonomy were used in subsequent analyses.

We generated Principal Coordinate Analysis (PCoA) using the phyloseq $\mathrm{R}$ package [54] from Bray-Curtis dissimilarity matrix after count normalization and $\log _{10}$ transformation using the following formula [55]:

$$
\log _{10}\left(\frac{R C}{n} \times \frac{\sum x}{N}+1\right)
$$


where $R C$ is the read count for a particular ASV in a particular sample, $n$ is the total number of reads in that sample, the sum of $x$ is the total number of reads in all samples and $N$ is the total number of samples. Family PCoA (Fig. 1b) was generated as described above except that ASVs were merged at the family level. For Fig. If and g, only taxa with a relative abundance $>1 \%$ are shown.

Difference in the microbial community composition (beta diversity) was tested using permutational multivariate analysis of variance (PERMANOVA) through the vegan $R$ package command adonis (version 2.5) with permutations set to 1000 .

Alpha diversity (Shannon and Simpson indices) was calculated using the phyloseq $\mathrm{R}$ package from the rarefied counts (samples rarefied to the minimum count in all samples: 33,516) and differences were tested using Wilcoxon rank sum test using $\mathrm{R}$ function wilcox.test.

Linear discriminant analysis effect size (LEfSe) [56] was used to identify biomarkers associated with Phase1 and Phase 2 and only OTUs with a $P$-value $<0.01$ and LDA threshold value $>3$ were considered a significant association.

\section{Metabolomics extraction and LC-MS}

A subset of neonate stool samples with additional available fecal material (term: $n=4$, pre-term: $n=5$ ) were used for further metabolomics analysis. Samples were suspended in $200 \mu \mathrm{L} 5 \mathrm{mM}$ Ammonium Acetate and homogenized thrice for $30 \mathrm{~s}$ each time using a cell disruptor. Protein concentrations of the homogenates were measured using a Qubit fluorometer and normalized to $500 \mu \mathrm{g} / \mathrm{mL}$ protein. Five microliter of internal standard mixture was spiked into each normalized sample. Extraction of metabolites was performed by protein precipitation by adding $200 \mu \mathrm{L}$ of extraction solution consisting of 8:1:1 Acetonitrile: Methanol: Acetone. Samples were mixed thoroughly, incubated at $4{ }^{\circ} \mathrm{C}$ to allow protein precipitation, and centrifuged at $20,000 \mathrm{~g}$. Supernatant was transferred into a new tube and dried using nitrogen. Samples were reconstituted with $25 \mu \mathrm{L}$ of reconstitution solution, mixed, and incubated at $4{ }^{\circ} \mathrm{C}$ for $10-15 \mathrm{~min}$. Samples were centrifuged at $20000 \times \mathrm{g}$ and supernatants collected for metabolomics profiling.

Global metabolomics profiling was performed on a Thermo Q-Exactive Orbitrap mass spectrometer with Dionex UHPLC and autosampler. All samples were analyzed in positive and negative heated electrospray ionization with a mass resolution of 35,000 at $\mathrm{m} / \mathrm{z} 200$ as separate injections. Separation was achieved on an ACE 18 -pfp $100 \times 2.1 \mathrm{~mm}, 2 \mu \mathrm{m}$ column with mobile phase $\mathrm{A}$ as $0.1 \%$ formic acid in water and mobile phase B as acetonitrile. The flow rate was $350 \mu \mathrm{L} / \mathrm{min}$ with a column temperature of $25^{\circ} \mathrm{C}$. Four microliter was injected for negative ions and $2 \mu \mathrm{L}$ for positive ions. Three technical replicates were performed per sample.

\section{Metabolomics analysis}

The mean of the three technical replicates was generated for each sample and used for analyses. Data were normalized by sum and log-transformed prior to analysis. Principal component analysis (PCA) was done using $\mathrm{R}$ prcomp function and difference between the two groups at $\mathrm{PC} 1$ was tested using Wilcoxon rank sum test using $\mathrm{R}$ function wilcox.test and $p$-values corrected for multiple comparisons using $\mathrm{R}$ function p.adjust using the method of Benjamini and Hochberg [57]. Data was further analyzed using Metaboanalyst software [58]. Only identified metabolites were included in these analyses. Metabolite analysis was conducted using the suggested mass tolerance of $0.25 \mathrm{~m} / \mathrm{z}$ and retention time of $30 \mathrm{~s}$, and filtered by interquartile range. Random forest was used to determine important metabolomics features using 500 trees. KEGG pathway analysis was conducted using the Metaboanalyst MS Peaks to Pathways module with a p-value cutoff of 0.01. Pathway enrichment was determined by adjusting normalized metabolite concentrations from pre-term samples relative to term samples, and significance assessed by Fisher's exact test.

We cross-referenced normalized identified metabolite concentrations from our samples with a list of known microbially-associated metabolites obtained from Kaiko et al. [21] and correlated their relative abundances with 16S rRNA sequencing data from the same samples. Pearson correlation coefficients were calculated using the $\mathrm{R}$ function cor.test and heatmaps generated using the $\mathrm{R}$ package pheatmap [59].

\section{Organoid preparation}

Small intestinal enteroids were generated from 4 to 8 week old wild type C57BL6 mice as previously described [51]. Enteroids were cultured in Advanced DMEM/F12 (Life Technologies 12,634) supplemented with $1 \mathrm{x} \mathrm{N}-2$ supplement (R\&D Systems AR009), 1x B27 supplement (Gibco 17, 504), $10 \mathrm{mM}$ HEPES (Thermo Fisher Scientific15630080), 1x Glutamax (Gibco 15,630,080), $100 \mathrm{U} / \mathrm{mL}$ PenicillinStreptomycin (Gibco 10,378,016), $50 \mathrm{ng} / \mathrm{mL}$ recombinant mouse EGF (Peprotech 315-09), $50 \mathrm{ng} / \mathrm{mL}$ recombinant murine noggin (Peprotech $250-38$ ), and $50 \mathrm{ng} / \mathrm{mL}$ recombinant mouse $r$-spondin CHO-expressed (R\&D Systems 7150-RS). Organoid cultures were passaged a minimum of one time prior to experimentation.

\section{Organoid proliferation}

Organoids were seeded in $10 \mu \mathrm{l}$ matrigel (Fisher Scientific 356,237 ) to 96 -well culture plates at a density of approximately 40 organoids per well and exposed to the pooled term $(n=4)$ and pre-term $(n=5)$ stool supernatant from the same samples used for metabolomics analysis. Supernatants were diluted at a 1:100 dilution for up to 5 days with daily media changes. Viability 
assays were conducted using the CellTiter 96 NonRadioactive Cell Proliferation Assay (Promega G4000) and absorbance was measured using a BioTek Synergy H4 Hybrid Microplate Reader. Measurements were normalized to mean absorbance values at plating to quantify changes in cell numbers. To measure organoid budding and area, approximately organoids (200 in $80 \mu \mathrm{l}$ matrigel) were seeded to 6-well culture plates and treated with stool samples at a 1:100 dilution for up to 5 days. A minimum of 5 representative images were taken per treatment, and a researcher blinded to experimental treatment used a random ROI generator to select organoids for morphological analysis using ImageJ.

\section{Immunofluorescent staining}

Mature, budding enteroids were passaged as previously described and treated by addition of stool supernatants at a 1:100 dilution for 3 days directly after passage. Organoids were collected, fixed, and permabilized as previously described [51] before incubation overnight at $4{ }^{\circ} \mathrm{C}$ with $0.5 \mu \mathrm{g} / \mathrm{ml} \mathrm{Ki67}$ antibody (Abcam \#ab15580), then for $1 \mathrm{~h}$ with Alexa Fluor 488 goat antirabbit secondary antibodies (Life Technologies \#A-11034). Specimens were then counterstained with 1:5000 DAPI solution (ThermoFisher \#62248) for $1 \mathrm{~h}$, mounted with Vectashield hard set mounting medium (Vector Laboratories \#H-1400) in a fluorodish (World Precision Instruments FD35-100) and imaged using a Leica TCS SP5 confocal microscope. Fluorescence was quantified using ImageJ to compare the total area of GFP stain relative to DAPI.

\section{Quantitative RT-PCR}

For qPCR analysis, enteroids were cultured in 6-well cultures plates as described above, treated for $48 \mathrm{~h}$ with stool supernatant, then collected and washed in cold PBS before resuspension in RLT buffer (Qiagen 217, 004). RNA was isolated from two wells using the RNeasy

Table 1 Quantitative RT-PCR Primer Sequences

\begin{tabular}{lll}
\hline Gene & 5'-3' Forward Primer & 3'-5' Reverse Primer \\
\hline Lgr5 & GCTTTGACACACATTCC & CTTCTCAAATTCTGTAGCG \\
Ascl2 & AAGCACACCTTGACTGGTACG & AAGTGGACGTTGCACCTTCA \\
Ephb2 & GCGGCTACGACGAGAACAT & GGCTAAGTCAAAATCAGCCTCA \\
Sox9 & CATCTCTCCTAATGCTATCTTC & CTGAGATTGCCCAGAGT \\
Fzd5 & CACGGTTGTCTTCCTCTTAGTC & ACCTGCGATGGCTTCATT \\
Lyz1 & GCCGATACTGGTGTAATG & CTCTCACCACCCTCTT \\
Muc2 & CCATCTCTACCACCATTAC & CTCGATCACCACCATTT \\
Chga & GTCCTGGAAGTCATCTC & AGTTCCTTCAGCAGATT \\
Chop & AGGAAACGAAGAGGAAGAA & CTGACTGGAATCTGGAGAG \\
TNF-a & ATGAGCACAGAAAGCATGATC & TACAGGCTTGTCACTCGAATT \\
IFNy & ACGCTTATGTTGTGCTGATGG & CTTCCTCATGGCTGTTCTGG \\
B-actin & TGGCGTGAGGGAGAGXXXAGX & AGCCATGTACGTAGCCATCCA \\
\hline
\end{tabular}

Miniprep Kit (Qiagen 217,004) and RT-PCR was performed using the iScript cDNA Synthesis Kit (Bio-Rad 170-8891). qPCR was performed using a Bio-RAD CFX384 Real Time PCR system using primers described in Table 1. RT-PCR was performed for 35 cycles with a $\mathrm{Tm}$ of $56^{\circ} \mathrm{C}$ and $\Delta \mathrm{Ct}$ values were calculated using the housekeeping gene $\beta$-actin. Fold change was calculated using the $-2^{\Delta \Delta \mathrm{Ct}}$ method [60].

\section{Statistics}

For organoid experiments, all statistical tests were performed using GraphPad Prism (version 6). The rest of the analyses were done using $\mathrm{R}$ [61] (version 3.4.4). All tests were two-tailed and determined statistically significant at $p<0.05$. Parametric tests were used to analyze normally distributed data, Mann-Whitney $U$ tests were used for all other data. For all enteroid experiments biological replication was achieved by pooling results from organoids isolated from unique mice.

\section{Supplementary information}

Supplementary information accompanies this paper at https://doi.org/10. 1186/s12866-020-01892-7.

Additional file 1: Supplemental Table 1. LC-MS data collected in positive ion mode. Raw data from term and pre-term LC-MS analysis including mass, retention time, and positive metabolite identification where available.

Additional file 2: Supplemental Table 2. LC-MS data collected in negative ion mode. Raw data from term and pre-term LC-MS analysis including mass, retention time, and positive metabolite identification where available.

Additional file 3: Supplemental Table 3. Relative abundance of identified metabolites. Relative abundances calculated from positive and negative ion modes. FDR corrected $p$-values for known metabolites between term and pre-term fecal samples.

Additional file 4: Supplemental Table 4. Enriched pre-term metabolic pathways mapped to Escherichia coli. A list of microbial metabolic pathways enriched in pre-term stool samples compared to term stool samples as determined by KEGG orthologs.

Additional file 5: Supplemental Table 5. Enriched pre-term metabolic pathways mapped to Homo sapiens. A list of human metabolic pathways enriched in pre-term stool samples compared to term stool samples as determined by KEGG orthologs.

\section{Abbreviations \\ ASV: Amplicon sequence variant; DCA: Deoxycholic acid; EGF: Epidermal growth factor; EGFR: Epidermal growth factor receptor; FXR: Farnesoid X receptor; GDCA: Glycodeoxycholic acid; KEGG: Kyoto Encyclopedia of Genes and Genomes; LAB: Lactic acid bacteria; LefSe: Linear discriminant analysis effect size; LC-MS: Liquid chromatography-mass spectrometry; NCBI: National Center for Biotechnology Information; NEC: Necrotizing enterocolitis; PC: Principle component; PCA: Principle component analysis; PCoA: Principal coordinate analysis; PERMANOVA: Permutational multivariate analysis of variance; PGE2: Prostaglandin E2; SRA: Sequence read archive; TCA: Tricarboxylic acid cycle; TUDCA: Tauroursodeoxycholic acid; UHPLC: Ultra-high-performance liquid chromatography}

\section{Acknowledgements}

The authors would also like to thank the UF Shands Pediatric Hospital for assistance with sample collection, and the Southeastern Center for Integrated Metabolomics for LC-MS analysis. 


\section{Authors' contributions}

Study concept and design (MWD, OK, MM, JN, CJ); Acquisition of data (MWD, OK); Analysis and interpretation of data (MWD, OK, RZG, CJ); Drafting of Manuscript (MWD, RZG, CJ); Statistical analysis (MWD, RZG); Obtained funding (MM, CJ); Study supervision (MM, JN, CJ). All authors had access to the study data and have reviewed and approved the final manuscript.

\section{Funding}

This research was supported by National Institutes of Health grants R01DK073338, University of Florida Health Cancer Center (UFHCC) Funds and University of Florida Department of Medicine Gatorade Fund all to CJ. RZG is supported by UFHCC funds.

\section{Availability of data and materials}

165 rRNA sequences have been uploaded to the National Center for Biotechnology Information Sequence Read Archive (NCBI SRA) under BioProject ID: PRJNA533302. All data generated or analyzed from global untargeted LC-MS are included in this published article and its supplementary information files. All other datasets used and/or analysed during the current study are available from the corresponding author on reasonable request.

\section{Ethics approval and consent to participate}

All experiments were performed in accordance with appropriate institutional guidelines. Collection of human neonatal stool samples were permitted under exempt protocol number 201801271 approved by the University of Florida Institutional Review Board. Consent for collection of neonatal fecal samples was waived by the University of Florida Institutional Review Board. Collection of murine small intestine for organoid generation was carried out under Institutional Animal Care and Use Committee protocol number 201608025.

\section{Consent for publication}

Not applicable.

\section{Competing interests}

The authors declare no competing interests.

\section{Author details}

'Department of Medicine, Division of Gastroenterology, University of Florida, CGRC, 2033 Mowry Rd, Florida 32610, USA. ${ }^{2}$ Department of Pediatrics, University of Florida, Gainesville, Florida, USA. ${ }^{3}$ Department of Infectious Diseases and Pathology, College of Veterinary Medicine, University of Florida, Gainesville, Florida, USA.

Received: 12 June 2019 Accepted: 2 July 2020

Published online: 11 July 2020

\section{References}

1. von Mutius E. The shape of the microbiome in early life. Nat Med. 2017; 23(3):274-5.

2. Moles L, Gómez M, Heilig H, Bustos G, Fuentes S, de Vos W, et al. Bacterial diversity in meconium of preterm neonates and evolution of their fecal microbiota during the first month of life. PLoS One. 2013;8(6):e66986.

3. Koenig JE, Spor A, Scalfone N, Fricker AD, Stombaugh J, Knight R, et al. Succession of microbial consortia in the developing infant gut microbiome. Proc Natl Acad Sci U S A. 2011;108(Suppl 1):4578-85.

4. Wampach L, Heintz-Buschart A, Fritz JV, Ramiro-Garcia J, Habier J, Herold M, et al. Birth mode is associated with earliest strain-conferred gut microbiome functions and immunostimulatory potential. Nat Commun. 2018;9(1):5091.

5. Grier A, Qiu X, Bandyopadhyay S, Holden-Wiltse J, Kessler HA, Gill AL, et al. Impact of prematurity and nutrition on the developing gut microbiome and preterm infant growth. Microbiome. 2017;5(1):158.

6. Dominguez-Bello MG, Costello EK, Contreras M, Magris M, Hidalgo G, Fierer $\mathrm{N}$, et al. Delivery mode shapes the acquisition and structure of the initial microbiota across multiple body habitats in newborns. Proc Natl Acad Sci U S A. 2010;107(26):11971-5.

7. Bäckhed F, Roswall J, Peng Y, Feng Q, Jia H, Kovatcheva-Datchary P, et al. Dynamics and stabilization of the human gut microbiome during the first year of life. Cell Host Microbe. 2015;17(5):690-703.
8. Neu J, Pammi M. Pathogenesis of NEC: impact of an altered intestinal microbiome. Semin Perinatol. 2017;41 (1):29-35.

9. Pammi M, Cope J, Tarr PI, Warner BB, Morrow AL, Mai V, et al. Intestinal dysbiosis in preterm infants preceding necrotizing enterocolitis: a systematic review and meta-analysis. Microbiome. 2017;5(1):31.

10. Mai V, Torrazza RM, Ukhanova M, Wang X, Sun Y, Li N, et al. Distortions in development of intestinal microbiota associated with late onset sepsis in preterm infants. PLoS One. 2013;8(1):e52876.

11. Diaz Heijtz. Fetal, neonatal, and infant microbiome: perturbations and subsequent effects on brain development and behavior. Semin Fetal Neonatal Med. 2016;21(6):410-7.

12. Bergmann KR, Liu SXL, Tian R, Kushnir A, Turner JR, Li H-L, et al. Bifidobacteria stabilize claudins at tight junctions and prevent intestinal barrier dysfunction in mouse necrotizing enterocolitis. Am J Pathol. 2013; 182(5):1595-606.

13. Stefka AT, Feehley T, Tripathi P, Qiu J, McCoy K, Mazmanian SK, et al. Commensal bacteria protect against food allergen sensitization. Proc Natl Acad Sci U S A. 2014;111(36):13145-50.

14. Hui S, Ghergurovich JM, Morscher RJ, Jang C, Teng X, Lu W, et al. Glucose feeds the TCA cycle via circulating lactate. Nature. 2017;551(7678):115-8.

15. Liu C, Wu J, Zhu J, Kuei C, Yu J, Shelton J, et al. Lactate inhibits lipolysis in fat cells through activation of an orphan G-protein-coupled receptor, GPR81. J Biol Chem. 2009;284(5):2811-22.

16. Lee Y-S, Kim T-Y, Kim Y, Lee S-H, Kim S, Kang SW, et al. Microbiota-derived lactate accelerates intestinal stem-cell-mediated epithelial development. Cell Host Microbe. 2018;24(6):833-846.e6.

17. Sonnenburg JL, Bäckhed F. Diet-microbiota interactions as moderators of human metabolism. Nature. 2016;535(7610):56-64.

18. Turnbaugh PJ, Hamady M, Yatsunenko T, Cantarel BL, Duncan A, Ley RE, et al. A core gut microbiome in obese and lean twins. Nature. 2009. 457(7228):480-4.

19. Den Besten G, van Eunen K, Groen AK, Venema K, Reijngoud D-J, Bakker BM The role of short-chain fatty acids in the interplay between diet, gut microbiota, and host energy metabolism. J Lipid Res. 2013;54(9):2325-40.

20. Metges CC. Contribution of microbial amino acids to amino acid homeostasis of the host. J Nutr. 2000;130(7):1857S-64S.

21. Kaiko GE, Ryu SH, Koues OI, Collins PL, Solnica-Krezel L, Pearce EJ, et al. The colonic crypt protects stem cells from microbiota-derived metabolites. Cell. 2016;165(7):1708-20.

22. Bastide P, Darido C, Pannequin J, Kist R, Robine S, Marty-Double C, et al. Sox 9 regulates cell proliferation and is required for Paneth cell differentiation in the intestinal epithelium. J Cell Biol. 2007;178(4):635-48.

23. McCoy KD, Ronchi F, Geuking MB. Host-microbiota interactions and adaptive immunity. Immunol Rev. 2017;279(1):63-9.

24. Kostic $A D$, Gevers $D$, Siljander $H$, Vatanen $T$, Hyötyläinen $T$, Hämäläinen $A-M$, et al. The dynamics of the human infant gut microbiome in development and in progression toward type 1 diabetes. Cell Host Microbe. 2015;17(2):260-73.

25. Solís G, de Los Reyes-Gavilan CG, Fernández N, Margolles A, Gueimonde M. Establishment and development of lactic acid bacteria and bifidobacteria microbiota in breast-milk and the infant gut. Anaerobe. 2010;16(3):307-10.

26. Shaffiey SA, Jia H, Keane T, Costello C, Wasserman D, Quidgley M, et al. Intestinal stem cell growth and differentiation on a tubular scaffold with evaluation in small and large animals. Regen Med. 2016;11(1):45-61.

27. Cherbuy C, Honvo-Houeto E, Bruneau A, Bridonneau C, Mayeur C, Duée P-H, et al. Microbiota matures colonic epithelium through a coordinated induction of cell cycle-related proteins in gnotobiotic rat. Am J Physiol Gastrointest Liver Physiol. 2010;299(2):G348-57.

28. Khoury KA. Small intestinal mucosal cell proliferation and bacterial flora in the conventionalization of the germfree mouse. J Exp Med. 1969;130(3): 659-70.

29. Bardocz S, Grant G, Brown DS, Pusztai A. Putrescine as a source of instant energy in the small intestine of the rat. Gut. 1998;42(1):24-8.

30. Janne J. Polyamines in rapid growth and cancer. Biochimica et Biophysica Acta (BBA) - Rev Cancer. 1978;473(3-4):241-93.

31. Morris DR, Pardee AB. A biosynthetic ornithine decarboxylase in Escherichia, coli. Biochem Biophys Res Commun. 1965;20(6):697-702.

32. Cunin R, Glansdorff N, Piérard A, Stalon V. Biosynthesis and metabolism of arginine in bacteria. Microbiol Rev. 1986;50(3):314-52.

33. Shaibe E, Metzer E, Halpern YS. Metabolic pathway for the utilization of Larginine, L-ornithine, agmatine, and putrescine as nitrogen sources in Escherichia coli K-12. J Bacteriol. 1985;163(3):933-7. 
34. Morris DR, Pardee AB. Multiple pathways of putrescine biosynthesis in Escherichia coli. J Biol Chem. 1966;241(13):3129-35.

35. Alifano P, Fani R, Liò P, Lazcano A, Bazzicalupo M, Carlomagno MS, et al. Histidine biosynthetic pathway and genes: structure, regulation, and evolution. Microbiol Rev. 1996;60(1):44-69.

36. Matsui $T$, Ichikawa H, Fujita T, Takagi T, Osada-Oka M, Minamiyama $Y$. Histidine deficiency attenuates cell viability in rat intestinal epithelial cells by apoptosis via mitochondrial dysfunction. J Nutr Intermed Metab. 2017:8:21-8.

37. Grandi D, Schunack W, Morini G. Epithelial cell proliferation is promoted by the histamine $H(3)$ receptor agonist (R)-alpha-methylhistamine throughout the rat gastrointestinal tract. Eur J Pharmacol. 2006;538(1-3):141-7.

38. Ridlon JM, Wolf PG, Gaskins HR. Taurocholic acid metabolism by gut microbes and colon cancer. Gut Microbes. 2016;7(3):201-15.

39. Martinez-Augustin O, Sanchez de Medina F. Intestinal bile acid physiology and pathophysiology. World J Gastroenterol. 2008;14(37):5630-40.

40. Inagaki T, Moschetta A, Lee Y-K, Peng L, Zhao G, Downes M, et al. Regulation of antibacterial defense in the small intestine by the nuclear bile acid receptor. Proc Natl Acad Sci U S A. 2006;103(10):3920-5.

41. Makishima M, Okamoto AY, Repa JJ, Tu H, Learned RM, Luk A, et al. Identification of a nuclear receptor for bile acids. Science. 1999: 284(5418):1362-5.

42. Fu T, Coulter S, Yoshihara E, Oh TG, Fang S, Cayabyab F, et al. FXR regulates intestinal cancer stem cell proliferation. Cell. 2019;176(5):1098-1112.e18.

43. Dong W, Liu L, Dou Y, Xu M, Liu T, Wang S, et al. Deoxycholic acid activates epidermal growth factor receptor and promotes intestinal carcinogenesis by ADAM17-dependent ligand release. J Cell Mol Med. 2018;22(9):4263-73.

44. Im E, Martinez JD. Ursodeoxycholic acid (UDCA) can inhibit deoxycholic acid (DCA)-induced apoptosis via modulation of EGFR/Raf-1/ERK signaling in human colon cancer cells. J Nutr. 2004;134(2):483-6.

45. Pai R, Tarnawski AS, Tran T. Deoxycholic acid activates beta-catenin signaling pathway and increases colon cell cancer growth and invasiveness. Mol Biol Cell. 2004;15(5):2156-63.

46. Xia G, Martin AE, Michalsky MP, Besner GE. Heparin-binding EGF-like growth factor preserves crypt cell proliferation and decreases bacterial translocation after intestinal ischemia/reperfusion injury. J Pediatr Surg. 2002;37(7):1081-7 discussion 1081

47. Jain U, Lai C-W, Xiong S, Goodwin VM, Lu Q, Muegge BD, et al. Temporal regulation of the bacterial metabolite Deoxycholate during colonic repair is critical for crypt regeneration. Cell Host Microbe. 2018;24(3):353-363.e5.

48. Connors J, Dunn KA, Allott J, Bandsma R, Rashid M, Otley AR, et al. The relationship between fecal bile acids and microbiome community structure in pediatric Crohn's disease. ISME J. 2019;14:702-13.

49. Afrazi A, Branca MF, Sodhi CP, Good M, Yamaguchi Y, Egan CE, et al. Tolllike receptor 4-mediated endoplasmic reticulum stress in intestinal crypts induces necrotizing enterocolitis. J Biol Chem. 2014;289(14):9584-99.

50. Srivillibhuthur M, Warder BN, Toke NH, Shah PP, Feng Q, Gao N, et al. TFAM is required for maturation of the fetal and adult intestinal epithelium. Dev Biol. 2018;439(2):92-101.

51. He Z, Gharaibeh RZ, Newsome RC, Pope JL, Dougherty MW, Tomkovich S, et al. Campylobacter jejuni promotes colorectal tumorigenesis through the action of cytolethal distending toxin. Gut. 2019;68(2):289-300.

52. Bolyen $\mathrm{E}$, Rideout JR, Dillon MR, Bokulich NA, Abnet C, Al-Ghalith GA, et al. QIIME 2: reproducible, interactive, scalable, and extensible microbiome data science; 2018.

53. Callahan BJ, McMurdie PJ, Rosen MJ, Han AW, Johnson AJA, Holmes SP. DADA2: high-resolution sample inference from Illumina amplicon data. Nat Methods. 2016;13(7):581-3.

54. McMurdie PJ, Holmes S. Phyloseq: an R package for reproducible interactive analysis and graphics of microbiome census data. PLoS One. 2013;8(4): e61217.

55. McCafferty J, Mühlbauer M, Gharaibeh RZ, Arthur JC, Perez-Chanona E, Sha W, et al. Stochastic changes over time and not founder effects drive cage effects in microbial community assembly in a mouse model. ISME J. 2013; 7(11):2116-25.

56. Segata N, Izard J, Waldron L, Gevers D, Miropolsky L, Garrett WS, et al. Metagenomic biomarker discovery and explanation. Genome Biol. 2011; 12(6):R60.

57. Benjamini Y, Hochberg Y. Controlling the false discovery rate: a practical and powerful approach to multiple testing. J R Stat Soc B. 1995;57(1):289-300.
58. Chong J, Soufan O, Li C, Caraus I, Li S, Bourque G, et al. MetaboAnalyst 4.0 towards more transparent and integrative metabolomics analysis. Nucleic Acids Res. 2018;46(W1):W486-94.

59. Kolde R. Pheatmap: pretty heatmaps. R package . R; 2015.

60. Livak KJ, Schmittgen TD. Analysis of relative gene expression data using real-time quantitative PCR and the 2(-Delta Delta C(T)) method. Methods. 2001;25(4):402-8.

61. R Core Team. R: a language and environment for statistical computing. Vienna: R Foundation for Statistical Computing; 2018.

\section{Publisher's Note}

Springer Nature remains neutral with regard to jurisdictional claims in published maps and institutional affiliations.
Ready to submit your research? Choose BMC and benefit from:

- fast, convenient online submission

- thorough peer review by experienced researchers in your field

- rapid publication on acceptance

- support for research data, including large and complex data types

- gold Open Access which fosters wider collaboration and increased citations

- maximum visibility for your research: over $100 \mathrm{M}$ website views per year

At BMC, research is always in progress.

Learn more biomedcentral.com/submissions 